\title{
(+)-Cholesten-3-one induces osteogenic differentiation of bone marrow mesenchymal stem cells by activating vitamin $D$ receptor
}

\author{
QIU-KE HOU ${ }^{1,2}$, YONG-QUAN HUANG ${ }^{3}$, YI-WEN LUO ${ }^{4}$, BIN WANG $^{4}$, YA-MEI LIU ${ }^{5}$, RU-DONG DENG ${ }^{1}$, \\ SAI-XIA ZHANG ${ }^{1}$, YING-TAO LAI ${ }^{2}$, WANG-YANG LI ${ }^{4}$ and DONG-FENG CHEN ${ }^{1}$ \\ ${ }^{1}$ Department of Anatomy, Guangzhou University of Traditional Chinese Medicine; \\ ${ }^{2}$ Department of Gastroenterology, The First Affiliated Hospital of Guangzhou University of Chinese Medicine; \\ ${ }^{3}$ Department of Orthopedics, The Second Affiliated Hospital of Guangzhou University of Traditional Chinese Medicine; \\ ${ }^{4}$ Department of Trauma, Orthopedics and Traumatology Hospital Affiliated to Guangzhou University of \\ Traditional Chinese Medicine; ${ }^{5}$ Department of Diagnosis of Traditional Chinese Medicine, Guangzhou \\ University of Traditional Chinese Medicine, Guangzhou, Guangdong 510000, P.R. China
}

Received October 25, 2015; Accepted November 25, 2016

DOI: $10.3892 /$ etm.2017.4200

\begin{abstract}
In our previous reports, it was revealed that steroids in traditional Chinese medicine (TCM) have the therapeutic potential totreatbonedisease.Inthepresentstudy, aninvitromodel of a vitamin D receptor response element (VDRE) reporter gene assay in mesenchymal stem cells (MSCs) was used to identify steroids that enhanced osteogenic differentiation of MSCs. (+)-cholesten-3-one $(\mathrm{CN})$, which possesses a ketone group that is modified in cholesterol and cholesterol myristate, effectively promoted the activity of the VDRE promoter. Phenotypic cellular analysis indicated that $\mathrm{CN}$ induced differentiation of MSCs into osteogenic cells and increased expression of specific osteogenesis markers, including alkaline phosphatase, collagen II and Runt-related transcription factor 2. Furthermore, CN significantly increased the expression of osteopontin, the target of the vitamin D receptor (VDR), which indicated that $\mathrm{CN}$ may activate vitamin $\mathrm{D}$ receptor signaling. Over-expression of VDR or knockdown studies with VDR-small interfering RNA revealed that the pro-differentiation effects induced by $\mathrm{CN}$ required VDR. Furthermore, the present study determined that the C-terminal region of the VDR is responsible for the action of $\mathrm{CN}$. Taken together, the present findings demonstrated that $\mathrm{CN}$ induced osteogenic differentiation of MSCs by activating VDR. The present study explored the regulation of stem cells by using a series of similar steroids and provided evidence to support a
\end{abstract}

Correspondence to: Professor Dong-Feng Chen, Department of Anatomy, Guangzhou University of Traditional Chinese Medicine, 232 Waihuan East Road, Guangzhou, Guangdong 510000, P.R. China

E-mail: cdf27212@21cn.com

Key words: (+)-cholesten-3-one, vitamin D receptor, mesenchymal stem cells, osteogenic differentiation potential strategy for the screening of novel drugs to treat bone disease in the future.

\section{Introduction}

Mesenchymal stem cells (MSCs) are mesodermal cells derived from bone marrow, which may differentiate into osteoblasts, chondrocytes, myoblasts, osteoblasts, chondrocytes, myoblasts, stromal cells, adipocytes and other cells (1). Dysregulation of the differentiation of MSCs into osteoblasts is involved in several bone-related diseases, including osteoporosis (2). MSCs have the potential to be used as therapeutic agents since they are easily isolated and extracted from patients, without ethical or technical problems. The inherent potential of MSCs makes them ideal seed cells for cell transplantation therapy, which is expected to become an effective treatment of intractable orthopedic disease $(3,4)$. Although MSCs are promising targets for the treatment of a number of bone diseases, the outcome of MSCs must be controlled before their therapeutic potential can be realized. Therefore, an improved understanding of the signaling pathways that control the differentiation of MSCs is required.

Nuclear receptors are ligand-activated transcription factors that perform a central function in the differentiation of stem cells (5). Among them, vitamin D receptor (VDR) has a critical role in the control of osteogenic differentiation of MSCs (6). VDR is composed of three distinct regions: An N-terminal region, containing a ligand-independent activation function-1; a highly conserved central region, containing the DNA binding domain; and the C-terminal region of the receptor, containing a multifunctional domain that harbors the ligand binding domain, the retinoid $\mathrm{X}$ receptor heterodimerization motif and ligand-dependent activation function-2 (7). VDR response elements (VDREs) have been identified in the promoters of vitamin D3-responsive genes, such as osteopontin (8). Previous studies have characterized MSCs as a target of VDR functions (9). Furthermore, the epigenetic regulation of the VDR may be key to rejuvenating 
osteoblastogenesis in MSCs from elders (10). The active form of vitamin $\mathrm{D}, 1 \alpha, 25$-dihydroxyvitamin $\mathrm{D} 3\left[1,25(\mathrm{OH})_{2} \mathrm{D} 3\right.$; VD3], is the ligand for VDR and has been revealed to enhance osteoblast-mediated mineralization (11). Moreover, vitamin $\mathrm{D}$ induced transcriptional networks in the osteoblast differentiation in a previous study (12). Thus, VDR is proposed as an important pharmacological target for signaling pathways involved in osteogenic differentiation of MSCs. Therefore, VDR can effectively screen for novel drugs from medicinal plants, particularly traditional Chinese herbs. We previously reported that steroids found in traditional Chinese herb were able to control the fate of MSCs $(13,14)$.

In the present study, an in vitro model was established using a VDRE reporter gene assay, which constructed a VDRE promoter reporter and was transfected into MSCs. Then MSCs were treated with steroids to identify VDR signaling activators that may be involved in the osteogenic differentiation. Furthermore, based on the previous finding that steroids are able to enhance osteogenic differentiation of MSCs (13), various structural analogs of steroids were investigated. The analogs included VD3, (+)-cholesten-3-one (CN), steraric acid ethyl ester (SE), cholesterol (CL), cholesterol myristate (CM), cyasterone (CE), $\beta$-sitosterol (SL) and oleanolic acid (OA). Among them, $\mathrm{CN}$ was the specific compound that most markedly promoted osteogenic differentiation of MSCs.

\section{Materials and methods}

Animal grouping and drug administration. A total of 10 male specific pathogen free Sprague-Dawley rats, aged 4 weeks (180-200 g), were acquired from the Animal Centre of Guangzhou University of Chinese Medicine (Guangzhou, China). All animals received humane care in accordance with the guidelines set out by the Care of Experimental Animals Committee of Guangzhou University of Chinese Medicine. Dulbecco's modified Eagle medium (DMEM) and fetal bovine serum (FBS) were purchased from Gibco (Thermo Fisher Scientific, Inc., Waltham, MA, USA); VDRE, VD3, dimethyl sulphoxide (DMSO) and other chemical reagents were purchased from Sigma-Aldrich (Merck Millipore, Darmstadt, Germany); alizarin red stain solution, alkaline phosphatase (ALP; BA0632), osteopontin (OPN; PB0589), Runt-related transcription factor 2 (RUNX2; BA3613-2), VDR (PB0479) and collagen II (BA0533) antibody were provided by Wuhan Boster Biological Technology, Ltd., (Wuhan, China); and SE, CL, CM, CN, CE, SL and OA were provided by Tokyo Chemical Industry Co., Ltd., (Tokyo, Japan).

Culture of MSCs. The femur and tibia were harvested from rats, anaesthetized by chloral hydrate $(330 \mathrm{mg} / \mathrm{kg}$; Wuhan Boster Biological Technology, Ltd.) and sacrificed by cervical dislocation, in order to collect fresh marrow. The marrow was mixed with complete medium (low glucose DMEM supplemented with $10 \% \mathrm{FBS}$ ) and gradient centrifuged at $900 \times \mathrm{g}$ for $30 \mathrm{~min}$ at room temperature with Percoll at a density of $1.073 \mathrm{~g} \bullet \mathrm{ml}^{-1}$. Cells of the appropriate density were collected, washed with PBS three times, manually counted using a light microscope and cultured at a density of $1 \times 10^{6} \mathrm{~cm}^{-2}$ on dishes supplemented with complete medium, in a humidified atmosphere at $37^{\circ} \mathrm{C}$ and $5 \% \mathrm{CO}_{2}$, using an incubator. The medium was refreshed and the suspension cells were removed every three days. Isolated MSCs were confluent after nine days, and were digested using $0.25 \%$ trypsin in order to promote separation. Cells were subsequently passaged at a density of $1 \times 10^{4} \mathrm{~cm}^{-2}$ onto dishes. MSCs that had been passaged three times were used for subsequent experiments. The surface antigen identification and differentiation ability of MSCs were verified by our previous study $(13,14)$.

Plasmids, small interfering (si) RNA cell transfection and assay for luciferase activity. The purchased pGL3-basic vector and VDRE were digested using purchased NheI and HindIII (Takara Biotechnology Co., Ltd., Dalian, China), and both were combined to generate the VDRE promoter-Luc vector. Plasmid for cytomegalovirus (CMV) was cotransfected to normalize the variations in transfection efficiency. The pGL3-basic vector, VDRE vector, and pCMV-Myc-basic, pCMV-Myc-VDR, pCMV-Myc-VDR-N and pCMV-Myc-VDR-C plasmids were provided by the National Laboratory of Medical Molecular Biology (Beijing, China).

The sequence of the siRNA oligonucleotide that was used to silence the VDR was 955-GUGCCAUUGAGGUCA UCAUTT-975. The scramble siRNA with random nucleotides was 5'-UUCUCCGAACGUGUCACGUTT-3' (negative control siRNA). Both oligonucleotides were synthesized by Genepharma Corp., (Shanghai, China). siRNA were transfected into cells using Lipofectamine ${ }^{\circledR} 2000$ (Invitrogen; Thermo Fisher Scientific, Inc.) according to the manufacturer's protocols.

Dual luciferase assay was performed to study the effects of VD3, SE, CL, CM, CN, CE, SL and OA on VDRE promoter activity. VD3 (30 $\left.\mathrm{ng} \cdot \mathrm{ml}^{-1}\right), \mathrm{SE}, \mathrm{CL}, \mathrm{CM}, \mathrm{CN}, \mathrm{CE}, \mathrm{SL}$ and OA were dissolved in DMSO, respectively, and subsequently added to MSCs with an increasing concentration of $0-100 \mu \mathrm{g} \cdot \mathrm{ml}^{-1}$. MSCs seeded in complete medium treated with an equal volume of DMSO without steroids served as the control group. Luciferase assays were performed using a Dual Luciferase Assay kit (Promega Corp., Madison, WI, USA) and were used to detect the luciferase activity. A total of $10 \mu \mathrm{l}$ of cell lysate was added to MSCs to detect firefly luciferase activity. Following the standardization of Renilla luciferase activity, the activity of Renilla luciferase was detected.

Immunocytochemistry. Following treatment with CN, MSCs were fixed with $4 \%$ polyoxymethylene for $20 \mathrm{~min}$ at room temperature and permeabilized with $0.25 \%$ Triton $\mathrm{X}-100$ (Sigma-Aldrich; Merck Millipore) and blocked with $0.1 \%$ bovine serum albumin (BSA; Roche Diagnostics, Basel, Switzerland). Subsequently, MSCs were incubated overnight at $4^{\circ} \mathrm{C}$ with the following primary antibodies at dilutions of 1:200: ALP, OPN, RUNX2 and collagen II. After washing three times with PBS, the cells were reacted with the horseradish peroxidase-conjugated secondary antibody (ab6721; 1:2,000; Abcam, Cambridge, $\mathrm{UK}$ ) for $30 \mathrm{~min}$ at $37^{\circ} \mathrm{C}$. The cell nuclei were stained with hematoxylin (Wuhan Boster Biological Technology, Ltd.) and visualized via light microscopy (Leica Camera AG, Wetzlar, Germany). Percentage of positive cells vs. total cells in 4 fields was calculated. Rat ALP, OPN, RUNX2 and collagen II primary antibodies (all 1:200), mixed with non-immune serum, were used to immunostain MSCs at $4^{\circ} \mathrm{C}$ overnight, respectively. Secondary 
A

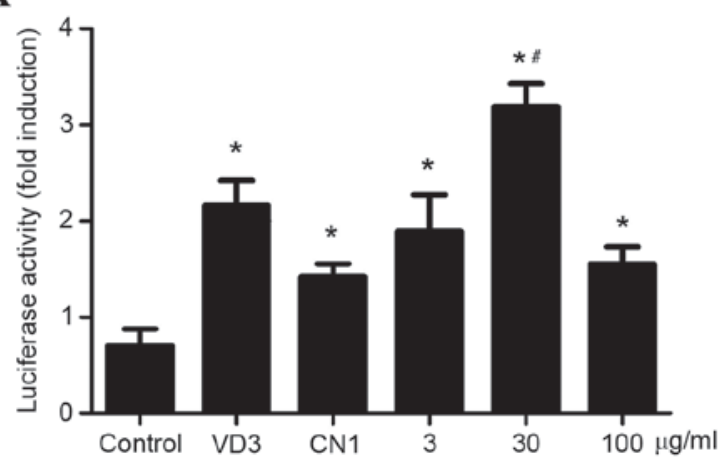

B

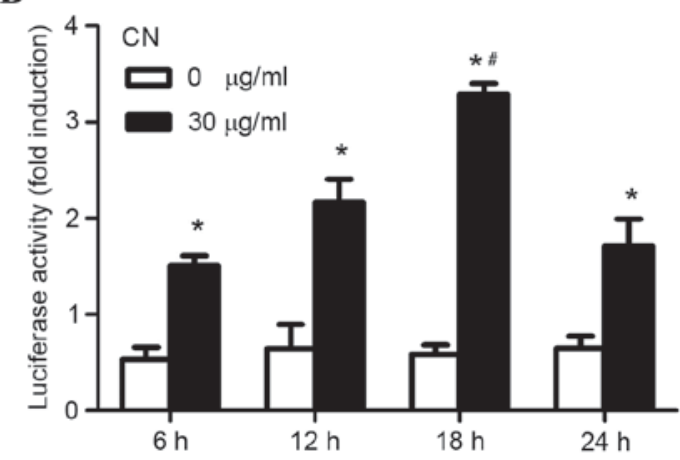

Figure 1. CN increases VDRE promoter activity. (A) Dose-response of $\mathrm{CN}$ on the VDRE promoter activity. ${ }^{*} \mathrm{P}<0.05$ vs. control; ${ }^{\sharp} \mathrm{P}<0.05$ vs. all other groups. (B) Time-response of $\mathrm{CN}$ on the VDRE promoter activity. ${ }^{*} \mathrm{P}<0.05 \mathrm{vs} .0 \mu \mathrm{g} / \mathrm{ml} ;{ }^{\#} \mathrm{P}<0.05$ vs. all other groups. All data were expressed as mean \pm standard deviation. $\mathrm{CN},(+)$-cholesten-3-one; VDRE, vitamin D response element.

antibody was used to incubate for $30 \mathrm{~min}$. The expression of ALP, OPN, RUNX2 and collagen II was detected after diaminobenzidine staining, followed by hematoxylin as the counterstain. Cytoplasm of positive cells were detected as deep brown. The same method was applied to the control group, except that the primary antibody with non-immune serum.

Calcium mineral deposition. MSCs were cultured for 14 days, as described. The level of calcium mineral deposition was revealed using alizarin-red staining (AR-S). Following three weeks in culture, cells were fixed with $70 \%$ ethanol, washed five times with deionized water and treated with $40 \mathrm{mM}$ alizarin-red solution for $10 \mathrm{~min}$ at $\mathrm{pH}$ 4.2. Subsequently, cells were washed with PBS for 15 min before being treated with $10 \%$ cetylpyridinium chloride in $10 \mathrm{mM}$ sodium phosphate for $15 \mathrm{~min}$ at room temperature. An AR-S standard curve, at an optical density of $540 \mathrm{~nm}$, was used to calculate the AR-S concentration.

Western blot analysis. Total protein was extracted from MSCs with cell lysis buffer (Invitrogen, Thermo Fisher Scientific, Inc.) and the protein concentration was determined using a Bradford protein assay (bicinchoninic acid protein assay kit; Bio-Rad Laboratories, Inc., Hercules, CA, USA). SDS-PAGE (6-15\%; Guge Biological Technology Co.) was created and separated proteins $(20 \mu \mathrm{g})$ were transferred to a membrane. The membrane was blocked in $5 \% \mathrm{BSA}$ at $24^{\circ} \mathrm{C}$ for $1 \mathrm{~h}$, incubated with primary polyclonal antibodies against ALP, OPN, RUNX2, VDR, collagen II (all 1:200) and $\beta$-catenin (ab32572; 1:5,000; Abcam, Cambridge, UK) at $4^{\circ} \mathrm{C}$ overnight, followed by three washes in TBST for 5 min each. The membrane was then incubated with Alexa Fluor-conjugated goat anti-rabbit IgG secondary antibody (ab150077; 1:2,000; Abcam) for $30 \mathrm{~min}$ at $24^{\circ} \mathrm{C}$, washed with TBST three times for $5 \mathrm{~min}$ each and protein bands were detected with DAB. The blot was scanned and optical density values of the targeted bands were analyzed with Image J software (1.48U; National Institutes of Health, Bethesda, MD, USA).

Statistical analysis. All data were expressed as mean \pm standard deviation. SPSS 20.0 software (IBM SPSS, Armonk, NY, USA) was used to analyze all data. $\mathrm{P}<0.05$ was considered to indicate a statistically significant difference.

\section{Results}

CN increases VDRE promoter activity. Induced expression of VDR provoked by CN stimulation of MSCs had been confirmed in our previous study $(13,14)$. The present study examined whether $\mathrm{CN}$ increased VDRE promoter activity. VDRE promoter reporter construct was transfected into MSCs and cells were treated with $1,3,30$ or $100 \mu \mathrm{g} \cdot \mathrm{ml}^{-1}$ of CN, respectively. MSCs that did not receive treatment were considered the control group, while VD3 was used for positive control, due to its effective promotion in VDRE promoter activity, as verified by a previous study. The highest VDRE promoter activity was observed at a CN concentration of $30 \mu \mathrm{g} \cdot \mathrm{ml}^{-1}$, which was statistically significant when compared to the other groups $(\mathrm{P}<0.05$; Fig. 1A). MSCs transfected with VDRE promoter reporter construct were treated with $30 \mu \mathrm{g} \cdot \mathrm{ml}^{-1}$ of $\mathrm{CN}$ and compared to MSCs that did not receive $\mathrm{CN}$ treatment. VDRE promoter activity was measured after $6,12,18$ and $24 \mathrm{~h}$ of exposure. The results indicated that the optimal time point of VDRE promoter activity of MSCs treated with $30 \mu \mathrm{g} / \mathrm{ml}$ of $\mathrm{CN}$ was $18 \mathrm{~h}$, as revealed by the significantly increased fold induction of luciferase activity $(\mathrm{P}<0.05$; Fig. 1B).

CN showed the highest activity on the VDRE, which may be associated with its ketone group. In our previous studies $(13,14)$, it was revealed that steroids had a stimulating effect on VDRE promoter activity; however, the specific compound responsible for achieving this effect is still unclear. The present study aimed to investigate MSCs that were transfected with VDRE promoter reporter construct, followed by either no treatment with the analogs of steroid (negative control), treatment with either VD3 (30 ng. $\mathrm{ml}^{-1}$, positive control), or with SE, CL, CM, $\mathrm{CN}, \mathrm{CE}, \mathrm{SL}$, and $\mathrm{OA}(30 \mu \mathrm{g} / \mathrm{ml})$ for $18 \mathrm{~h}$ (Fig. 2A). A significantly increased activity level was observed in CN-treated MSCs when compared with MSCs treated with the other steroids $(\mathrm{P}<0.05$; Fig. 2B). This indicated that $\mathrm{CN}$ was the optimal compound in promoting the highest VDRE promoter activity, even higher than VD3, which was the positive control $(\mathrm{P}<0.05)$. Compared with the other compounds, especially $\mathrm{CL}$ and SL with high similarity with $\mathrm{CN}$, the highest activity was observed in CN-treated MSCs; however, the only structural difference between $\mathrm{CN}$ and $\mathrm{CL}$ is the presence of a ketone group and $\mathrm{OH}$ group, respectively, which suggested that the 
A<smiles>C=C1CCC[C@@H](O)C[C@H]1/C=C1\CCC[C@]2(C)C1CCC2C(C)CCCC(C)C</smiles>

$1,25(\mathrm{OH})_{2} \mathrm{D} 3$

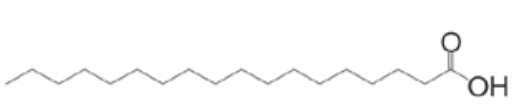

Steraric acid ethyl ester

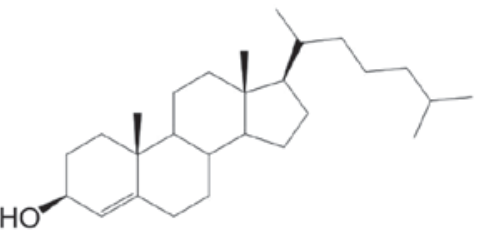

Cholesteol

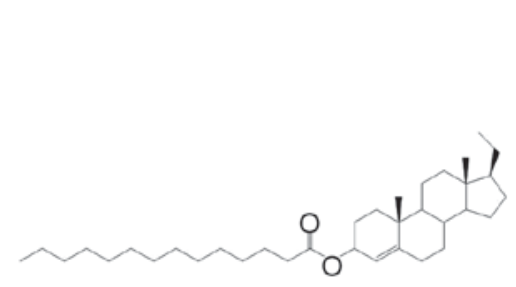

Cholesteol myristate<smiles>CC(C)CCCC(C)C1CCC2C3CCC4=CC(=O)CCC4(C)C3CCC12C</smiles>

(+)-cholesten-3-one

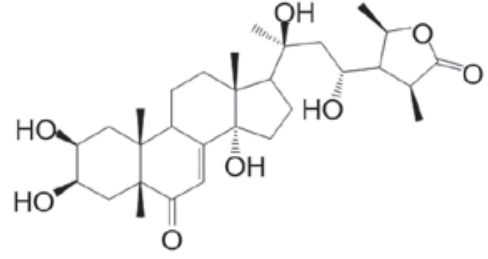

Cyasterone<smiles>CCC(CCC(C)C1CCC2C3CCC4CC(O)CCC4(C)C3CCC12C)C(C)C</smiles>

$\beta$-sitosterol

B

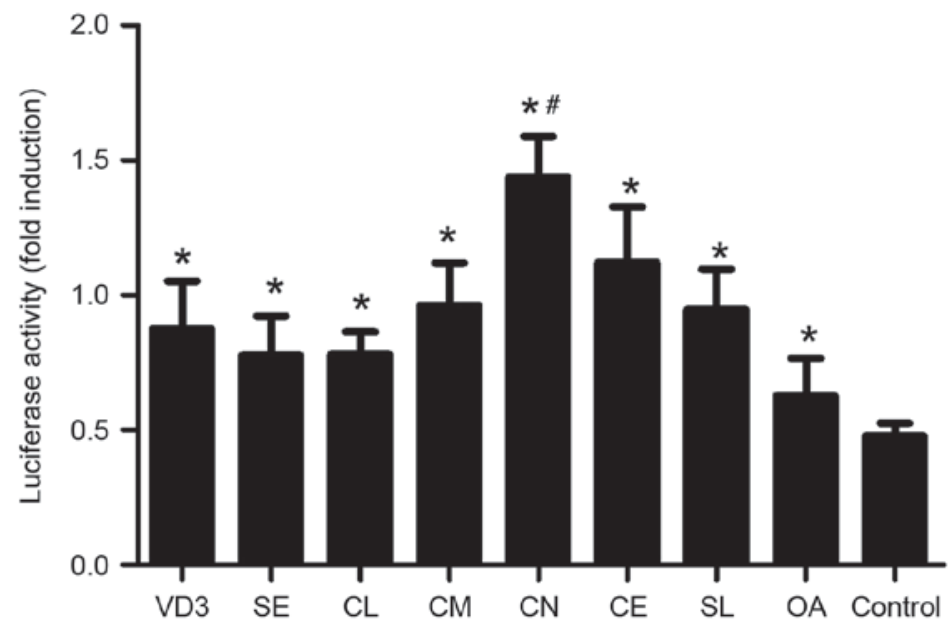

Figure 2. Effective activity of VDRE promoter is associated with the ketone group of CN that promotes the effective activity of the VDRE promoter. (A) Structure of VD3, SE, CL, CM, CN, CE, SL and OA. (B) Comparative effects of VD3, SE, CL, CM, CN, CE, SL and OA. All data were expressed as mean \pm standard deviation. "P $<0.05$ vs. control; ${ }^{*} \mathrm{P}<0.05$ vs. all other groups. CN, VD3, 1 $\alpha$, 25-dihydroxyvitamin D3; SE, steraric acid ethyl ester; CL, cholesterol; CM, cholesterol myristate; CE, cyasterone; SL, $\beta$-sitosterol; OA, oleanolic acid.

ketone group of $\mathrm{CN}$ may be involved in promoting VDRE promoter activity.

CN promotes osteogenic differentiation of MSCs. MSCs were stimulated by $\mathrm{CN}(30 \mu \mathrm{g} / \mathrm{ml})$ for seven days, immunocytochemically stained using osteogenesis markers and AR-S and further analyzed using western blotting. Immunocytochemical staining for osteogenesis markers containing ALP, OPN, RUNX2 and collagen II indicated that $\mathrm{CN}$ promoted osteogenic differentiation of MSCs as CN-treated MSCs exhibited a significantly increased number of osteogenisis-positive cells compared with MSCs that did not receive $\mathrm{CN}$ treatment ( $\mathrm{P}<0.05$; Fig. 3A). AR-S demonstrated that $\mathrm{CN}$-treated MSCs exhibited an increased number of calcium mineral deposition derived from osteoblasts when compared with MSCs that were not treated with CN (Fig. 3B). Furthermore, western blotting revealed significantly higher 
A
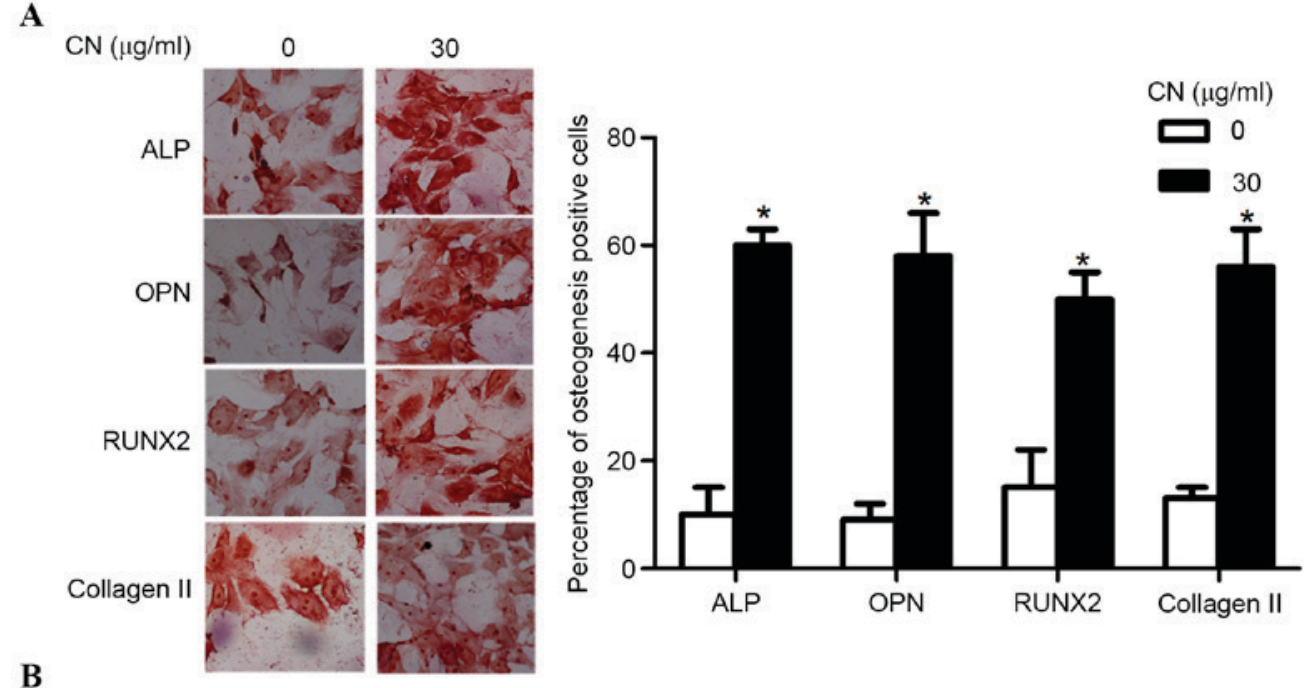

B
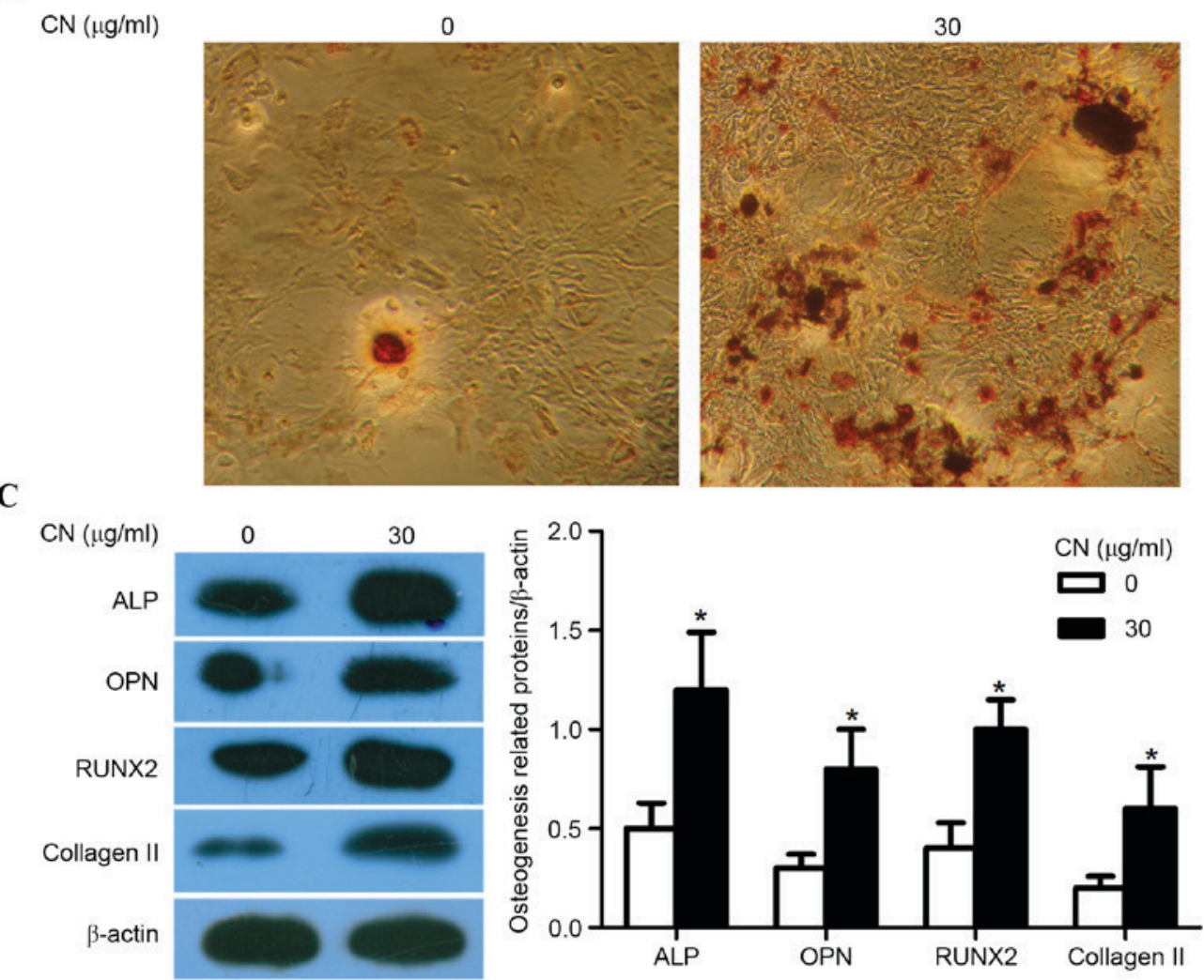

Figure 3. CN promotes the osteogenic differentiation of MSCs. (A) Photomicrographs were captured at a magnification of x200 and demonstrated the levels of ALP, OPN, RUNX2 and collagen II expressed in MSCs, where the cytoplasm was stained dark brown (left panel). Comparison of the percentage of ALP, OPN, RUNX2 and collagen II-positive cells after CN treatment for seven days (right panel). (B) Alizarin-red staining revealed the calcium mineral deposition of $\mathrm{CN}$-treated MSCs compared with MSCs that were not treated with $\mathrm{CN}$ dyed by alizarin-red staining. (C) Western blot analysis indicated the expression of ALP, OPN, RUNX2 and collagen II (left panel) by comparing the relative density of ALP/ $\beta$-actin, OPN/ $\beta$-actin, RUNX2/ $\beta$-actin and collagen II/ $\beta$-actin band (right panel). All data were expressed as mean \pm standard deviation. "P<0.05 vs. $0 \mu \mathrm{g} / \mathrm{ml}$. MSCs, mesenchymal stem cells; ALP, alkaline phosphatase; OPN, osteopontin; RUNTX2, runt-related transcription factor 2; CN, (+)-cholesten-3-one.

expression of ALP, OPN, RUNX2 and collagen II in CN-treated MSCs when compared to MSCs that did not receive CN treatment, indicating that $\mathrm{CN}$-treated MSCs exhibited significantly increased osteogenic cell differentiation $(\mathrm{P}<0.05 ;$ Fig. 3C).

CN promotes osteogenic differentiation of MSCs and requires $V D R$. MSCs transfected with VDR cDNA construct were treated with optimal concentrations of $\mathrm{CN}$. The expression of OPN and VDR were significantly increased in the group that was comprised of the combination of VDR cDNA and $\mathrm{CN}$ compared with the group where VDR cDNA and CN were both absent. $(\mathrm{P}<0.05$; Fig. 4A). MSCs were also transfected with a VDR siRNA construct and treated with optimal concentrations of CN. Furthermore, VDR siRNA inhibited the expression of OPN and VDR in MSCs compared with the groups where VDR siRNA was present and $\mathrm{CN}$ was absent $(\mathrm{P}<0.05$; Fig. 4B). These experiments indicated that $\mathrm{CN}$-induced differentiation of MSCs required VDR. 
A
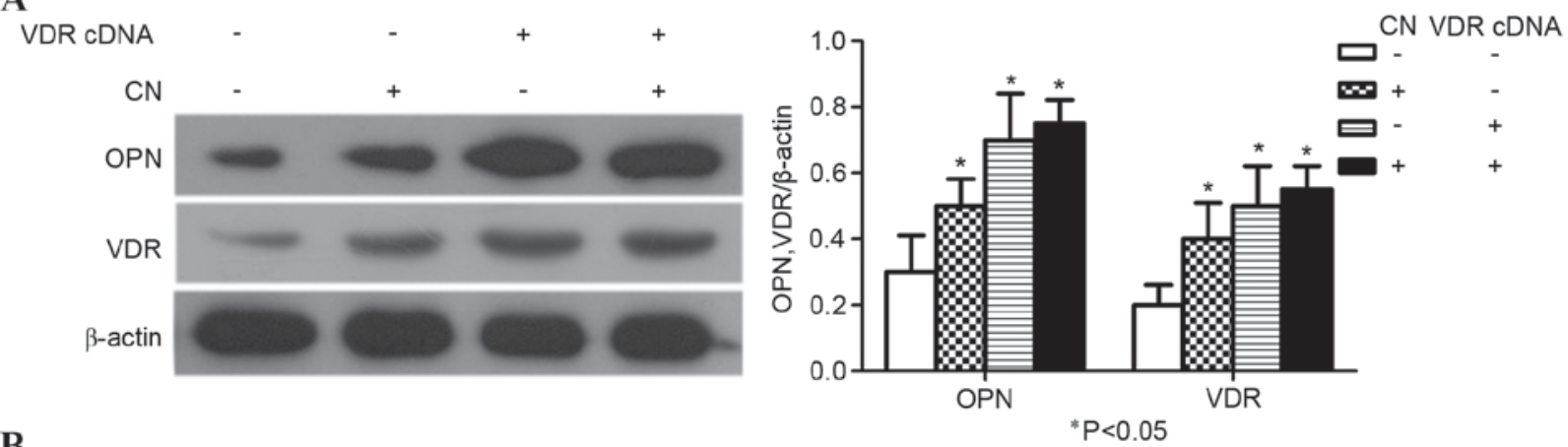

B
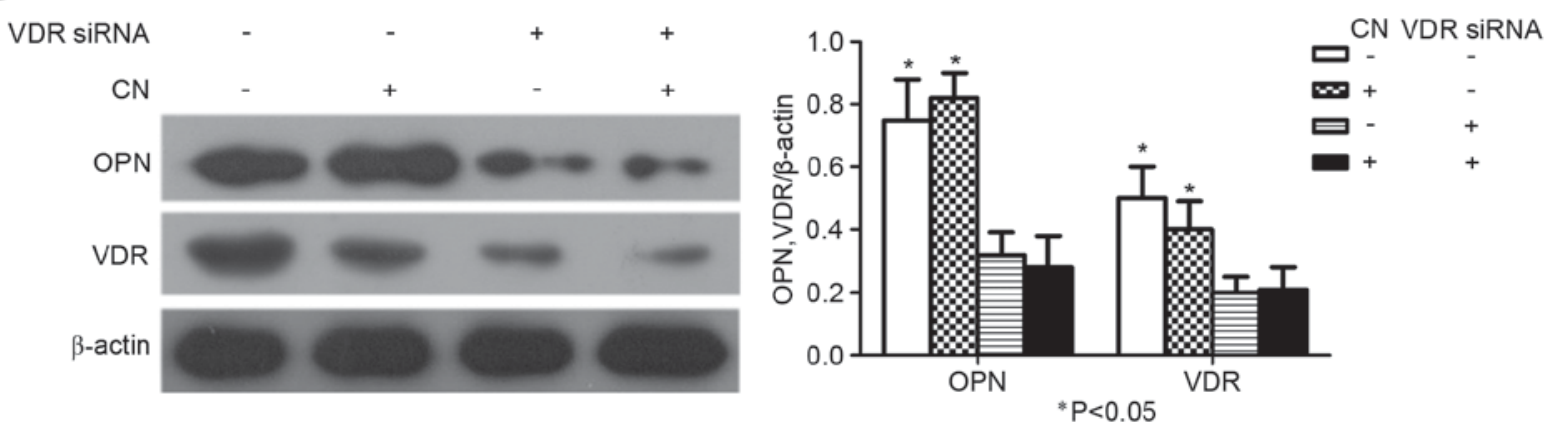

Figure 4. CN promotes the osteogenic differentiation of MSCs that require VDR. (A) Western blot analysis of OPN and VDR expressed in MSCs, treated with VDR cDNA construct and CN (left panel); comparison of the relatively density of OPN, VDR/actin band (right panel). "P<0.05 vs. CN and VDR cDNA were absent. (B) Western blot analysis of OPN and VDR expressed in MSCs, treated with VDR siDNA construct and CN (left panel); comparison of the relatively density of OPN, VDR/actin band (right panel). "P $<0.05$ vs. CN was absent and VDR siRNA was present. All data were expressed as mean \pm standard deviation. MSCs, mesenchymal stem cells; VDR, vitamin D receptor; OPN, osteopontin; siRNA, small interfering RNA; CN, (+)-cholesten-3-one.

$C$-terminal region of VDR is responsible for the action of $C N$. VDR fusion proteins included the following: VDR 1-91, an N-terminal region containing activation function-1 (AF-1) domain and a DNA binding domain (DBD) and VDR 115-427, a C-terminal region with a ligand binding domain, and a ligand-dependent AF-2 domain (Fig. 5A). To confirm whether $\mathrm{CN}$ interaction with the binding domains of VDR affected the differentiation of MSCs into osteogenic cells, MSCs were stimulated by $\mathrm{CN}$ and transfected with either pCMV-Myc-basic, pCMV-Myc-VDR, pCMV-Myc-VDR-N or pCMV-Myc-VDR-C plasmids. After seven days, immunocytochemical staining demonstrated that groups transfected with pCMV-Myc-VDR-C plasmids exhibited significantly increased osteogenic-positive cells $(\mathrm{P}<0.05$; Fig. 5B and $\mathrm{C})$. Western blot analysis indicated the expression of ALP, OPN and RUNX2 increased when MSCs were treated with $\mathrm{CN}$ and transfected with pCMV-Myc-VDR-C plasmids (Fig. 6A and B). Moreover, AR-S identified that CN-treated MSCs transfected with pCMV-Myc-VDR-C plasmids exhibited increased numbers of osteogenic-positive cells when compared with the positive and negative control groups (Fig. 6C).

\section{Discussion}

MSCs are capable of self-renewal and differentiation into osteogenic cells; however, the efficiency of these functions is typically low (15) and the process is not fully understood. Thus, the present study aimed to elucidate the process of MSC differentiation into osteogenic cells by exploring its physiological mechanism and therapeutic potential. In the present study, a functional stem cell assay was used based on the
VDR pathway as this facilitated improved understanding of the identified compounds involved in this pathway. The major findings of the present study were: i) $\mathrm{CN}$ exhibited significantly higher VDRE promoter activity when compared with SE, CL, $\mathrm{CM}, \mathrm{CE}$, SL and OA; ii) CN-promoted osteogenic differentiation of MSCs that required VDR; and iii) the C-terminal region of the VDR is involved in the action of $\mathrm{CN}$. The present study demonstrated a model that revealed structure-function relationships between steroids and VDR. The findings of the present study provide the incentive to develop a pioneering strategy to screen novel drugs for orthopedic disorders and to study a series of similar compounds involved in the regulation of stem cells.

In the present study, $\mathrm{CN}$ was identified as an inducer of MSC differentiation into osteoblasts, through cell phenotypic analysis. MSCs differentiate into all mesodermal cell types, including osteoblasts, chondrocytes, myoblasts, stromal cells and skeletal muscle cells, under appropriate conditions. Therefore, MSCs were chosen as a cell model in the present study. There is growing interest in the use of MSCs for the repair of bone damage; however, this requires efficient protocols for directing the differentiation of MSCs into the osteoblast lineage. Several studies have explored the use of bone morphogenetic proteins to induce MSCs toward the osteoblast lineage $(16,17)$. Furthermore, these reports have described the positive effect of large molecules on the osteogenesis of MSCs; however, small molecules that may promote the osteogenic differentiation of MSCs have not been identified $(16,17)$. Previous findings have also indicated that dexamethasone and VD3 were used to promote MSCs differentiation in vitro (18). In the present study, immunohistochemical analysis and 
A

\begin{tabular}{|l|l|}
\hline \multicolumn{2}{|c|}{24} \\
\hline AF-1 & DBD \\
\hline AF-1 & DBD \\
\hline
\end{tabular}

$\operatorname{VDR}_{115-427}$

$91 \quad 115$

115

$409 \quad 427$

\begin{tabular}{|l|l|l|}
\hline Hinge & LBD & AF-2 \\
\hline
\end{tabular}

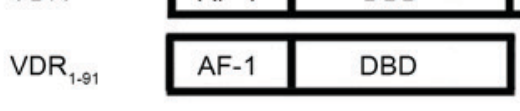

(1)

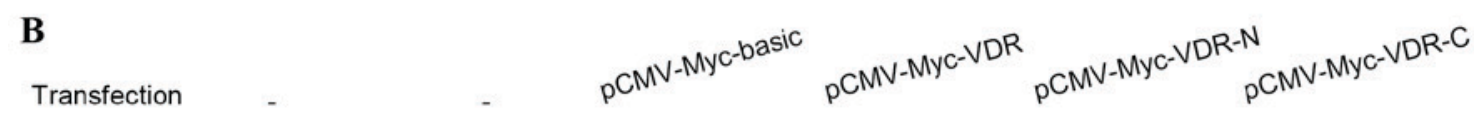

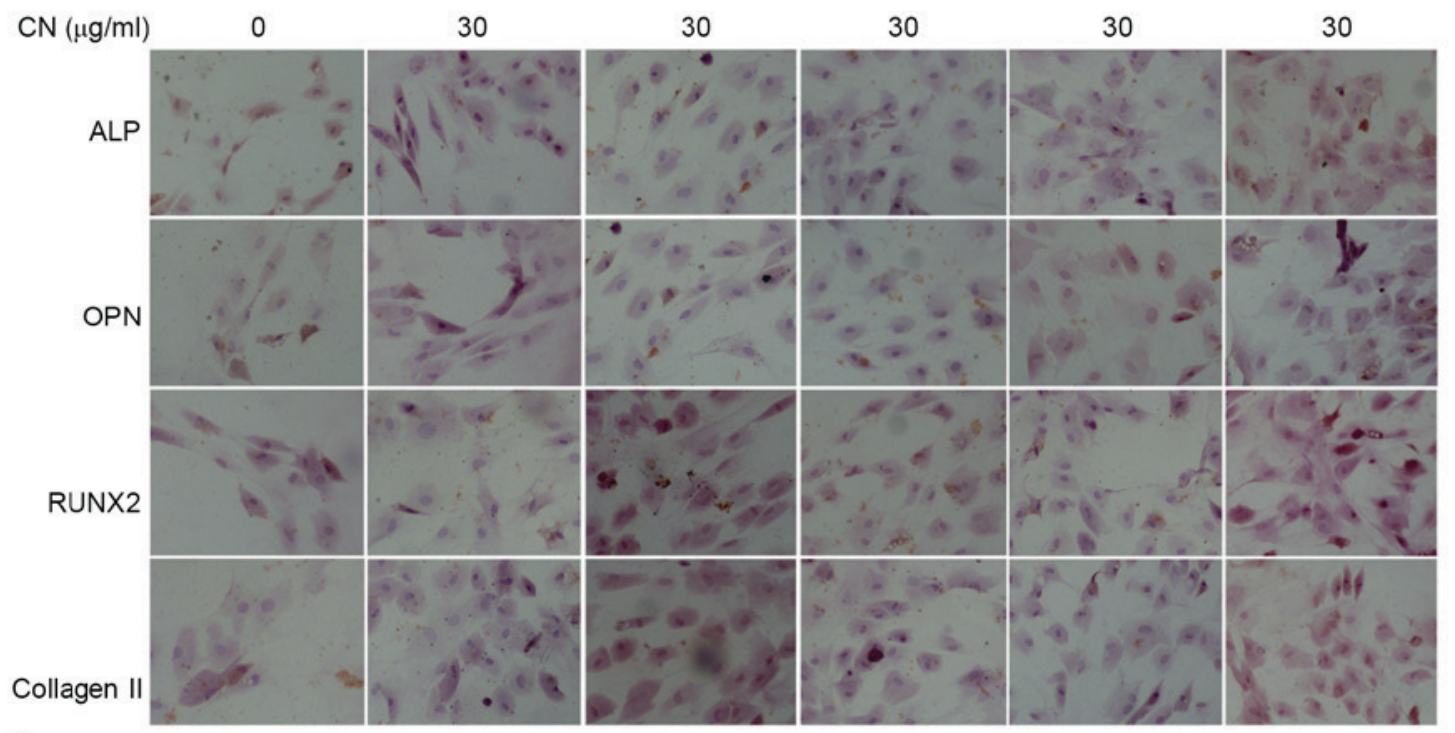

C

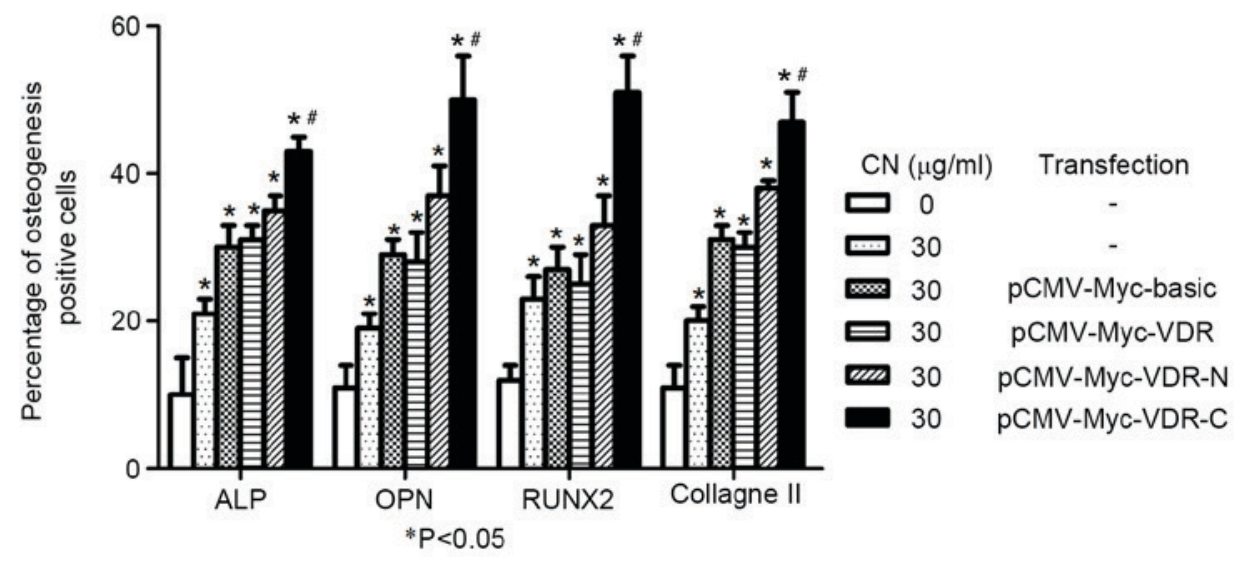

Figure 5. C-terminal region of the VDR is responsible for the action of CN. (A) Structure of VDR. (B) Photomicrographs showing ALP, OPN, RUNX2 and collagen II-positive cells in cytoplasm, which were dark brown (magnification, x200). (C) Comparison of the percentage of ALP, OPN, RUNX2 and collagen II-positive cells after $\mathrm{CN}$ treatment and transfection for seven days. All data were expressed as mean \pm standard deviation. ${ }^{*} \mathrm{P}<0.05$ vs. CN and transfection were absent; ${ }^{\mathrm{P}}<0.05$ vs. all other groups. VDR, vitamin D receptor; OPN, osteopontin; CN, (+)-cholesten-3-one; ALP, alkaline phosphatase; RUNX2, runt-related transcription factor 2; CMV, cytomegalovirus.

western blot analysis established that CN induced MSCs to differentiate into osteoblasts. This result is consistent with our previous findings, which provided evidence that $\mathrm{CN}$ promotes the differentiation of neural stem cells (19). Therefore, CN may be a potential therapeutic molecule for treating differentiation-related bone diseases.

Another notable finding of the present study was that $\mathrm{CN}$ functions as an activator of VDR. VDR, which consists of an N-terminal DNA-binding domain, a C-terminal ligand-binding domain and an intervening hinge region, is the nuclear hormone receptor of the vitamin D endocrine system that is predominantly involved in the maintenance of calcium and phosphate homeostasis and bone development (20-22). Furthermore, VDR is a ligand-induced nuclear transcription factor that regulates the expression of genes in critical mineral-regulating target tissues, such as bone, in order to maintain appropriate mineral homeostasis. Humans and mouse models have indicated that a lack of functional VDR is associated with severe bone diseases (20-22). Furthermore, it has also been suggested that VDR has a direct role in promoting osteoblast differentiation (23). Additionally, prior reports have identified that VDR-null mice exhibited a reduction in osteoblasts, accompanied by a decrease in trabecular bone volume (24), and MSC differentiation was impaired in 
A

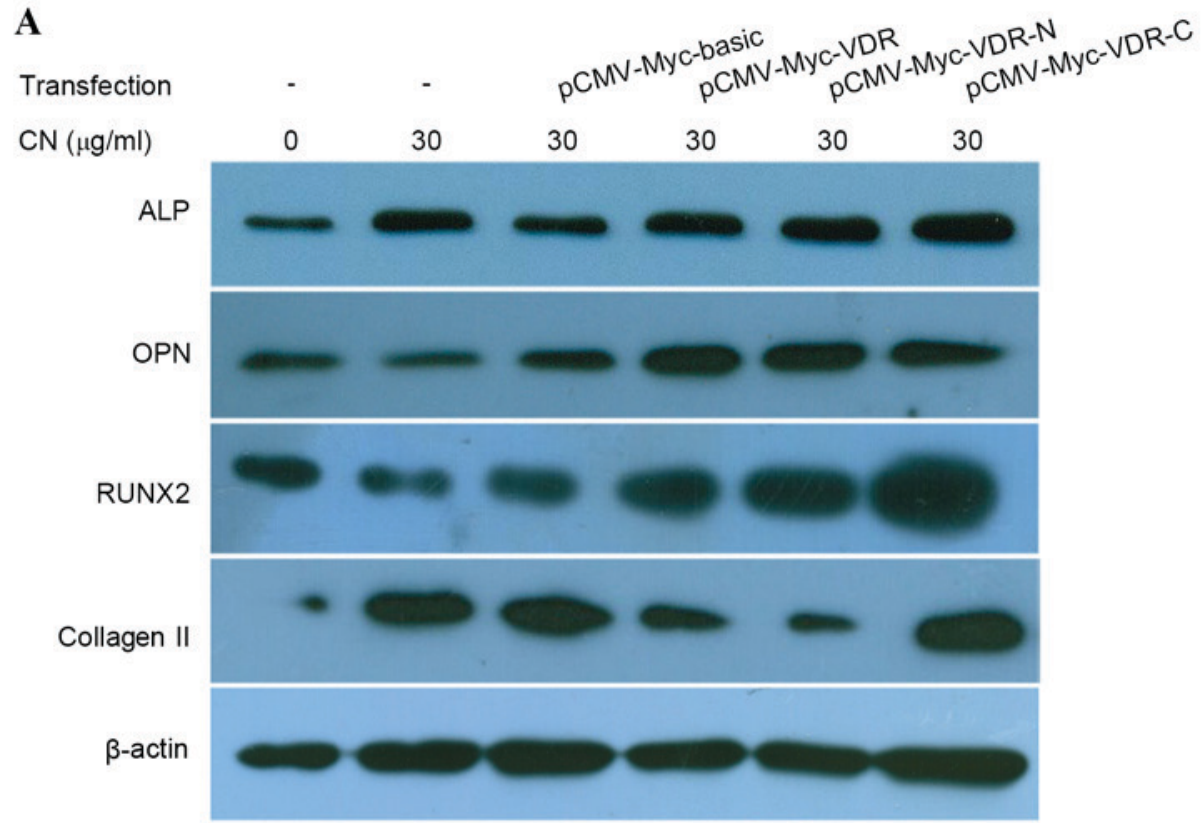

B

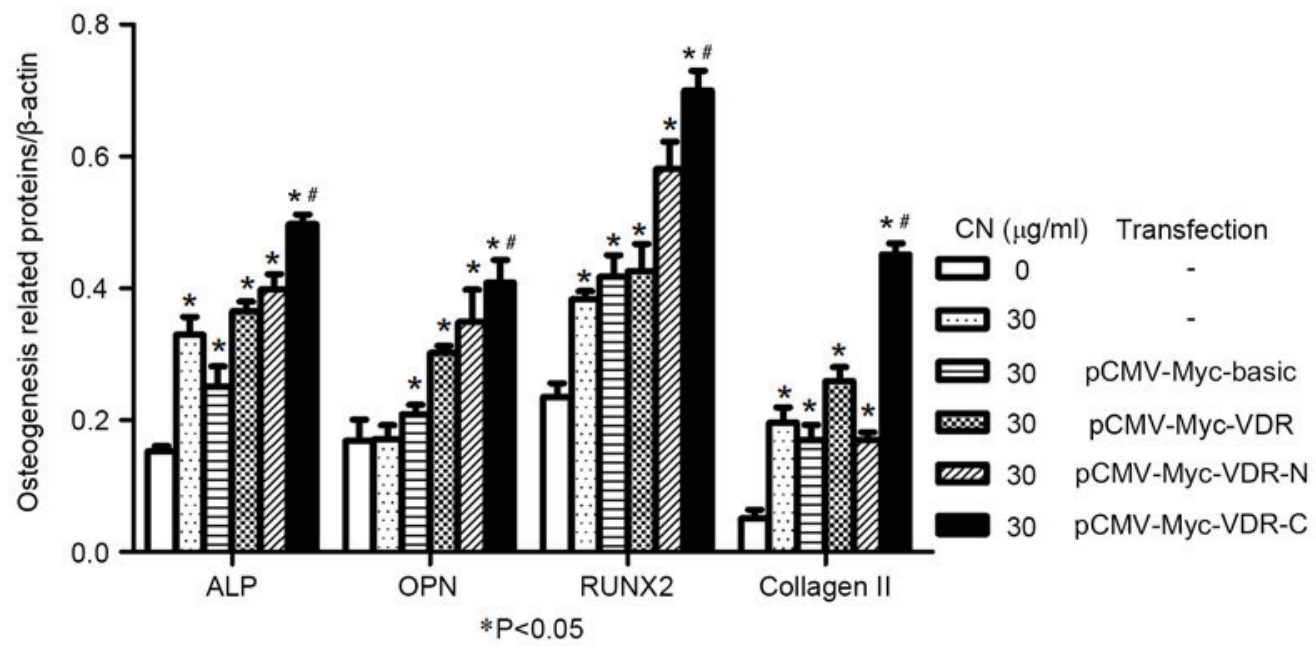

C

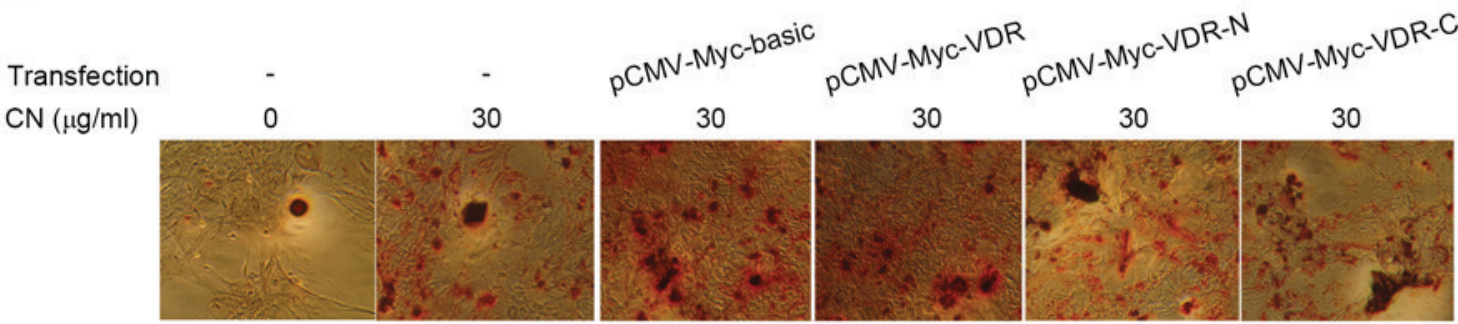

Figure 6. Western blot and alizarin-red staining established that the C-terminal region of the VDR is associated with activity. (A) Western blotting revealed the levels of ALP, OPN, RUNX2 and collagen II expressed in MSCs after transfection. (B) Comparison of the density between the experimental groups. (C) AR-S indicated the level of calcium mineral deposition. All data were expressed as mean \pm standard deviation. ${ }^{*} \mathrm{P}<0.05$ vs. $\mathrm{CN}$ and transfection were absent. " $\mathrm{P}<0.05$ vs. all other groups. MSCs, mesenchymal stem cells; VDR, vitamin D receptor; OPN, osteopontin; ALP, alkaline phosphatase; RUNTX2, runt-related transcription factor 2 .

VDR mice that presented with haploinsufficiency (25). Thus, strategies targeting VDR pathways for the stimulation of MSC differentiation are highly desirable.

We hypothesized that VDR may be one of the direct mediators of CN actions. Several experiments were performed to test this hypothesis, including the use of transcription assays, which employed a VDRE-firefly luciferase reporter plasmid and immunoblotting of CN-mediated induction of VDR target genes. Furthermore, over-expression of VDR and knockdown studies with VDR-siRNA were performed, in addition to a series of deletion mutants of the VDR and a MSCs differentiation assay. Notably, compared with the other investigated compounds, $\mathrm{CN}$ showed the highest promotion for the activity of VDRE promoter; but CL and SL with the higher similarity with CN, exhibited reduced VDRE promoter activity. This indicated that the ketone group of $\mathrm{CN}$ may be a functional group that is associated with the activity of VDRE. The present study also revealed that $\mathrm{CN}$ induced the expression of the VDR target genes, such 
as osteopontin, which suggested that the VDR pathway was activated by $\mathrm{CN}$. This result is consistent with observations on the activity of the VDRE promoter from the present study. siRNA-mediated knockdown of VDR in the MSCs differentiation model system revealed that the pro-differentiation effects of $\mathrm{CN}$ required VDR. Furthermore, a series of deletion mutants of the VDR were generated and used to identify that the C-terminal region of the VDR is responsible for the action of $\mathrm{CN}$. These results are consistent with previous reports that nuclear receptors are activated by small lipophilic ligands $(5,7,9)$.

The present findings have promising clinical applications. With the prevalent-use of $\mathrm{CN}$, it may be possible to improve the quality of engineered bone via MSC-mediated osteogenesis. This is an example of pharmacological control of MSC-mediated osteogenesis and highlights the need for an extensive screen of other families of small molecules both for bone formation and to regulate other differentiation pathways. Manipulation of MSC-mediated osteogenesis is an interesting alternative approach as the agents used in vitro were cleared from the graft before implantation.

VDR is an important target for osteoporosis and targeting VDR pharmacologically for the purpose of modulating stem cell function is a promising area in regenerative medicine. By identifying the role of VDR in MSCs and developing corresponding specific ligands as modulators, the therapeutic delivery of stem cells may become more controlled and efficient. Hence, the activation of vitamin $\mathrm{D}$ receptor by $\mathrm{CN}$ may provide a novel concept for the treatment of bone diseases, such as fracture repair and osteoporosis. Finally, systemic administration of $\mathrm{CN}$ may be used to treat patients by targeting either their endogenous or donated MSCs.

In conclusion, the present study demonstrated that a stem cell analysis system based on VDR signaling may be used to identify molecules that induce the osteogenic differentiation of MSCs and specifically revealed that CN significantly increased the efficiency of osteogenic differentiation of MSCs by activating the vitamin $\mathrm{D}$ receptor. These findings may have various applications in the field of bone and joint repair and raise interesting questions about the role of $\mathrm{CN}$ in osteogenesis.

\section{Acknowledgements}

The present study was supported by the National Natural Science Foundation of China (grant nos. 81273896 , 81273783 and 81473699), Guangdong Technology Projects of Self-financing Category (grant no. 2014807), Guangxi Scientific and Technological Issues (grant no. 2015AD09631) and Research Projects of Construction of Chinese Medicine province of Bureau of Traditional Chinese Medicine of Guangdong Province (grant no. 20141084).

\section{References}

1. Pittenger MF, Mackay AM, Beck SC, Jaiswal RK, Douglas R, Mosca JD, Moorman MA, Simonetti DW, Craig S and Marshak DR: Multilineage potential of adult human mesenchymal stem cells. Science 284: 143-147, 1999.

2. Olsen BR, Reginato AM and Wang W: Bone development. Annu Rev Cell Dev Biol 16: 191-220, 2000.

3. Cavallo C, Desando G, Cattini L, Cavallo M, Buda R, Giannini S, Facchini A and Grigolo B: Bone marrow concentrated cell transplantation: Rationale for its use in the treatment of human osteochondral lesions. J Biol Regul Homeost Agents 27: 165-175, 2013.
4. Gao C, Seuntjens J, Kaufman GN, Tran-Khanh N, Butler A, Li A, Wang H, Buschmann MD, Harvey EJ and Henderson JE: Mesenchymal stem cell transplantation to promote bone healing. J Orthop Res 30: 1183-1189, 2012.

5. Gronemeyer H, Gustafsson JA and Laudet V: Principles for modulation of the nuclear receptor superfamily. Nat Rev Drug Discov 3: 950-964, 2004.

6. Olivares-Navarrete R, Sutha K, Hyzy SL, Hutton DL, Schwartz Z, McDevitt T and Boyan BD: Osteogenic differentiation of stem cells alters vitamin D receptor expression. Stem Cells Dev 21: 1726-1735, 2012.

7. Giguere V: Orphan nuclear receptors: From gene to function Endocr Rev 20: 689-725, 1999.

8. Noda M, Vogel RL, Craig AM, Prahl J, DeLuca HF and Denhardt DT: Identification of a DNA sequence responsible for binding of the 1,25-dihydroxyvitamin D3 receptor and 1,25-dihydroxyvitamin D3 enhancement of mouse secreted phosphoprotein 1 (SPP-1 or osteopontin) gene expression. Proc Natl Acad Sci USA 87: 9995-9999, 1990.

9. Jeong Y and Mangelsdorf DJ: Nuclear receptor regulation of stemness and stem cell differentiation. Exp Mol Med 41: 525-537, 2009.

10. Zhou S, Geng S and Glowacki J: Histone deacetylation mediates the rejuvenation of osteoblastogenesis by the combination of $25(\mathrm{OH}) \mathrm{D} 3$ and parathyroid hormone in MSCs from elders. J Steroid Biochem Mol Biol 136: 156-159, 2013.

11. Woeckel VJ, Bruedigam C, Koedam M, Chiba H, van der Eerden BC and van Leeuwen JP: 1 $\alpha, 25$-dihydroxyvitamin D3 and rosiglitazone synergistically enhance osteoblast-mediated mineralization. Gene 512: 438-443, 2013.

12. van de Peppel J and van Leeuwen JP: Vitamin D and gene networks in human osteoblasts. Front Physiol 5: 137, 2014.

13. Chen DF, Zeng HP, Du SH, Li H, Zhou JH, Li YW, Wang TT and Hua ZC: Extracts from Plastrum testudinis promotes proliferation of rat bone-marrow derived mesenchymal stem cells. Cell Prolif 40: 196-212, 2007.

14. Chen DF, Zhang HL, Du SH, Li H, Zhou JH, Li YW, Zeng HP and Hua ZC: Cholesterol myristate suppresses the apoptosis of mesenchymal stem cells via upregulation of inhibitor of differentiation. Steroids 75: 1119-1126, 2010

15. Meijer GJ, de Bruijn JD, Koole R and van Blitterswijk CA: Cell-based bone tissue engineering. PLoS Med 4: e9, 2007.

16. Li X and Cao X: BMP signaling and skeletogenesis. Ann N Y Acad Sci 1068: 26-40, 2006.

17. Ryoo HM, Lee MH and Kim YJ: Critical molecular switches involved in BMP-2-induced osteogenic differentiation of mesenchymal cells. Gene 366: 51-57, 2006.

18. Siddappa R, Fernandes H, Liu J, van Blitterswijk C and de Boer J: The response of human mesenchymal stem cells to osteogenic signals and its impact on bone tissue engineering. Curr Stem Cell Res Ther 2: 209-220, 2007.

19. Chen DF, Meng LJ, Du SH, Zhang HL, Li H, Zhou JH, Li YW, Zeng HP and Hua ZC: (+)-Cholesten-3-one induces differentiation of neural stem cells into dopaminergic neurons through BMP signaling. Neurosci Res 68: 176-184, 2010.

20. Bouillon R, Carmeliet G, Verlinden L, van Etten E, Verstuyf A, Luderer HF, Lieben L, Mathieu C and Demay M: Vitamin D and human health: Lessons from vitamin D receptor null mice. Endocr Rev 29: 726-776, 2008

21. Verstuyf A, Carmeliet G, Bouillon R and Mathieu C: Vitamin D: A pleiotropic hormone. Kidney Int 78: 140-145, 2010.

22. Gallagher JC and Sai AJ: Vitamin D insufficiency, deficiency, and bone health. J Clin Endocrinol Metab 95: 2630-2633, 2010.

23. Zhou S, Glowacki J, Kim SW, Hahne J, Geng S, Mueller SM, Shen L, Bleiberg I and LeBoff MS: Clinical characteristics influence in vitro action of 1,25-dihydroxyvitamin $\mathrm{D}(3)$ in human marrow stromal cells. J Bone Miner Res 27: 1992-2000, 2012.

24. Panda DK, Miao D, Bolivar I, Li J, Huo R, Hendy GN and Goltzman D: Inactivation of the 25-hydroxyvitamin D 1alpha-hydroxylase and vitamin $\mathrm{D}$ receptor demonstrates independent and interdependent effects of calcium and vitamin D on skeletal and mineral homeostasis. J Biol Chem 279: 16754-16766, 2004.

25. de Paula FJ, Dick-de-Paula I, Bornstein S, Rostama B, Le P, Lotinun S, Baron R and Rosen CJ: VDR haploinsufficiency impacts body composition and skeletal acquisition in a gender-specific manner. Calcif Tissue Int 89: 179-191, 2011. 